\title{
Rubost Team Formation Control for Quadrotors
}

\author{
Wesam Jasim and Dongbing Gu
}

\begin{abstract}
In this work, we compare between $H_{\infty}$ and Integral Backstepping (IBS) controllers, while they applied to a leaderfollower formation problem of quadrotors. The controllers were used to control the quadrotors with external disturbances and model parameter uncertainties consideration. Nonlinear $H_{\infty}$ design approach is introduced for a general nonlinear affine system to derive a robust controller by solving a Hamilton-Jacobi inequality. Then robustness conditions of the proposed controller are derived via selecting an appropriate parametrised Lyapunov function. The resultant state feedback controller establishes the asymptotically stability of the closed-loop nonlinear system. IBS controller is derived and the stability analysis is achieved via Lyapunov function. Simulation results show a good performance for both controllers in normal circumstance and $H_{\infty}$ controller perform much better than IBS controller in disturbances circumstance. Experimental results of using $H_{\infty}$ controller show its stability and robustness against the disturbances.
\end{abstract}

\section{INTRODUCTION}

In last decade, the focus of control single unit quadrotor has expanded to control a team of quadrotors to be able to achieve their tasks in variable weather and complicated environments. Team formation flight also provide advantages over the use of an individual quadrotor in both civil and military applications, such as inspection of inaccessible area, disaster management, and search and rescue in risky circumstances, etc. Most of these applications demand more than one quadrotor to accomplish the desired objective [1]-[3]. The leader-follower approach is one of the main approaches of formation control design.

Distributed and decentralised control techniques were used in the literature to solve the leader-follower control problem. The distributed control technique assumes that not all followers receive the leader's information and there is a kind of cooperation among them [4]-[14], while the decentralised control technique proposes that all followers are able to receive the leader's information [15]-[20].

Different controllers have been implemented with both distributed and decentralized control techniques. In [4] the problem of the leader-follower consensus of a swarm of rigid body space crafts system was analysed based on quaternion representation using a distributed control technique. They assumed that the communication between two neighbouring followers is bidirectional and that all followers can receive the leader information. Stability analysis was obtained via Lyapunov theory and the simulation results proved the attitude and angular velocity tracking stability. A nonlinear control theory was presented to ensure the stability of quadrotors team formation in [9]. The wireless networks communication among the team was obtained via medium access control protocols.

The authors are with School of Computer Science and Electronic Engineering, University of Essex, Wivenhoe Park, Colchester, UK. wmjasi@essex.ac.uk, dgu@essex.ac.uk
Experimental tests verified the proposed algorithm with time delay consideration. An integrated optimal control framework was presented in [10] for the formation of a group of UAVs problem. The methodology was to integrate the formation control, trajectory tracking, and obstacle avoidance into one optimal control framework.

A robust LQR controller was proposed for individual quadrotors and team formation as well in [8]. The controller was designed for a linearised system around the hovering point. The simulation results indicated the ability of the controller to overcome the changes in communication topology among the robots with no dynamic effects. A NNs controller was presented in [5] for addressing the leader-follower problem. These two studies used Lyapunov theory to analyse the controller stability.

A backstepping controller was discussed in [11] based on graph theory to maintain the distance among the robots and in [7] with balanced graph and strong connection among the robots. The quadrotors' dynamic systems were linearised around the hovering point and a good performance was obtained in normal circumstances. A distributed coordinated control scheme was proposed by [6] to solve the problem of time-delay in leader-follower team formation communication of quadrotors and the simulation results under sufficient conditions demonstrated the validity of the presented control technique. A distributed cohesive motion control scheme was presented in [12] for 3D motion to maintain the distance among robots. This technique was developed to become a decentralised technique and significant attempts to deal with decentralised control techniques have been made. In [13] a MPC technique with integrated trajectory planning was analysed with a planning horizon for both team formation and obstacle avoidance. The method showed good simulation results. Again the MPC technique was presented in [14] to solve the problem of dynamic encirclement for a group of UAVs. The comtroller was tested in simulation and experiment. The simulation and experimental results show the ability of the controller to guide the vehicles to the desired radius.

On the other hand, some controllers have been proposed with decentralized control technique. For instance, a hybrid supervisory control based on a polar partitioning approach was suggested in [15] for the team formation problem and for collision avoidance as well. The combination of discrete quadrotors dynamic system and the supervisor was achieved using the parallel composition and the simulation results displayed that this method allows the supervisors to achieve a free collision in normal environments. A MPC technique was proposed in [20], where its hierarchical control effectiveness was compared with the potential field technique. The stability of the feedback controller based on fluid dynamic models in [16] was obtained based on smoothed-particle hydrodynamic. 
The simulation results of the above methods validated the proposed approaches.

Authors in [17] proposed the trajectory planners and feedback controllers for following the planned trajectory. Next they proposed a nonlinear decentralised controller for an aggressive formation problem in the micro quadrotors team in [18]. Communication failures and network time delays impact on team formation efficiency were considered. Local information of neighbour robots in the team was used for individual trajectory planning. Preserving the required form was based on the status estimation of neighbour robots. Then the authors presented two approaches to overcome the problem of concurrent assignment and planning of trajectories (CAPT) for the quadrotors team, a decentralised D-CAPT and centralised C-CAPT in [19]. The decentralised D-CAPT and centralised C-CAPT results were compared in simulation and practice and the experimental results demonstrated a good performance in indoor application.

With regard to the leader-follower control problem, Abdessameud and Tayebi [21] proposed a procedure which depends on a quaternion representation and is split up into translational and rotational control design under the upper bounded translational control input. Analysis of the closedloop system stability was achieved using Lyapunov theory. The proposed strategy took 8 seconds to catch the desired formation shape. In [22] a human user for teleoperation with a haptic device was proposed for the quadrotor team formation control problem with the cooperation of a backstepping controller. The simulation results revealed the ability of the human user to teleoperate in order to perform the formation. A triangle formation control of three quadrotors using optimal control techniques via the Pontryagin maximum principle was presented in [23] and the simulation results showed the effectiveness of using team formation rather than using an individual quadrotor in terms of fuel consumption. In [24] a consensus problem of swarm systems was discussed to obtain the time-varying formation based on double-integrator system modelling. The experimental results of the three quadrotors in formation verified the effectiveness of the proposed approach in dynamic-free conditions.

The results in most of the previous papers on leader-follower formation control of multi-quadrotor system did not consider the effect of external disturbances, such as payload changes (or mass changes), wind disturbance, inaccurate model parameters, etc., which often affected the quadrotors' control performance. Therefore, a quadrotor controller must be robust enough in order to reject the effect of disturbances and cover the change in model parameter uncertainties and external disturbances.

$H_{\infty}$ control approach is able to attenuate the disturbance energy by measuring a ratio between the energy of input signals and the energy of disturbance signals [25]. In this paper, we focus on using integral backstepping controller for the team formation control problem. The obtained results is then compared with the original $H_{\infty}$ controller controller. This paper is organised as follows, Section II introduces the formation control platform used in this work and its dynamics. Section III describes the details of state feedback controllers for the leader and followers. Section IV presents the integral backstepping controller derivation. In section $\mathrm{V}$ a comparison between the two controllers are illustrated via simulation results. Section VI provides experimental results. Our conclusion and future work are given in Section VII.

\section{LEADER-FOLLOWER FORMATION PROBLEM}

\section{A. Quadrotor Model}

To describe the orientation of a quadrotor, the Euler representation is used. The full dynamic model of a quadrotor can be written as:

$$
\left\{\begin{array}{l}
\dot{\mathbf{p}}_{i}=\mathbf{v}_{i} \\
\dot{\mathbf{v}}_{i}=-g \mathbf{e}+\frac{f_{i}}{m_{i}} R_{i} \mathbf{e} \\
\dot{\zeta}_{i}=\eta_{i} \\
J_{i} \dot{\eta}_{i}=S\left(\eta_{i}\right) J_{i} \eta_{i}+G\left(\eta_{i}\right)-\tau_{i}
\end{array}\right.
$$

where $i$ is $L$ for the leader and $F$ for the follower, $m_{i}$ is the quadrotor mass, $\zeta_{i}=\left[\varphi_{i}, \theta_{i}, \varphi_{i}\right]^{T}, \eta_{i}=\left[\dot{\varphi}_{i}, \dot{\theta}_{i}, \dot{\varphi}_{i}\right]^{T}, J_{i}$ is the $3 \times 3$ diagonal matrix representing three inertial moments in the body frame, $G\left(\eta_{i}\right)$ represents the gyroscopic effect, $\tau_{i}$ is the torque vector applied on the quadrotor, $\mathbf{v}_{i}=\left[v_{i x}, v_{i y}, v_{i z}\right]^{T}$ is the linear velocity, $\mathbf{p}_{i}=\left[x_{i}, y_{i}, z_{i}\right]^{T}$ is the position vector, the vector $\mathbf{e}=[0,0,1]^{T}$, and the rotation matrix $R_{i}$ is:

$R_{i}=\left[\begin{array}{ccc}c \psi_{i} c \theta_{i} & c \psi_{i} s \theta_{i} s \varphi_{i}-s \psi_{i} c \varphi_{i} & c \psi_{i} s \theta_{i} c \varphi_{i}+s \psi_{i} s \varphi_{i} \\ s \psi_{i} c \theta_{i} & s \psi_{i} s \theta_{i} s \varphi_{i}+c \psi_{i} c \varphi_{i} & s \psi_{i} s \theta_{i} c \varphi_{i}-c \psi_{i} s \varphi_{i} \\ -s \theta_{i} & c \theta_{i} s \varphi_{i} & c \theta_{i} c \varphi_{i}\end{array}\right]$

\section{B. Leader-follower Formation Control Problem}

One leader and one follower are considered in the leaderfollower formation control problem to be solved in this paper. The leader control problem is formulated as a trajectory tracking, and the follower control problem is also formulated as a tracking problem, but with a different tracking target.

The follower keeps its yaw angle $\left(\psi_{F}\right)$ the same as the leader when it maintains the formation pattern. It moves to a desired position $\mathbf{p}_{F d}$, which is determined by a desired distance $d$, a desired incidence angle $\rho$, and a desired bearing angle $\sigma$. A new frame $F^{\prime}$ is defined by the translation of the leader frame $L$ to the frame with the desired follower position $\mathbf{p}_{F d}$ as the origin. As shown in Figure 1, the desired incidence angle is measured between the desired distance $d$ and the $x-y$ plane in the new frame $F^{\prime}$, and the desired bearing angle is measured between the $x$ axis and the projection of the $d$ in $x-y$ plane in the new frame $F^{\prime}$. The desired position $\mathbf{p}_{F d}$ is

$$
\mathbf{p}_{F d}=\mathbf{p}_{L}-R_{L}^{T} d\left[\begin{array}{c}
\cos \rho \cos \sigma \\
\cos \rho \sin \sigma \\
\sin \rho
\end{array}\right]
$$

Now, the formation control problem for the follower is to satisfy the following conditions:

$$
\left\{\begin{array}{l}
\lim _{t \rightarrow \infty}\left(\mathbf{p}_{F d}-\mathbf{p}_{F}\right)=0 \\
\lim _{t \rightarrow \infty}\left(\psi_{L}-\psi_{F}\right)=0
\end{array}\right.
$$

The leader just tracks a desired trajectory represented by $\left(\mathbf{p}_{L d}, \psi_{L d}\right)$. So, the formation control problem for the leader is to satisfy the following conditions: 


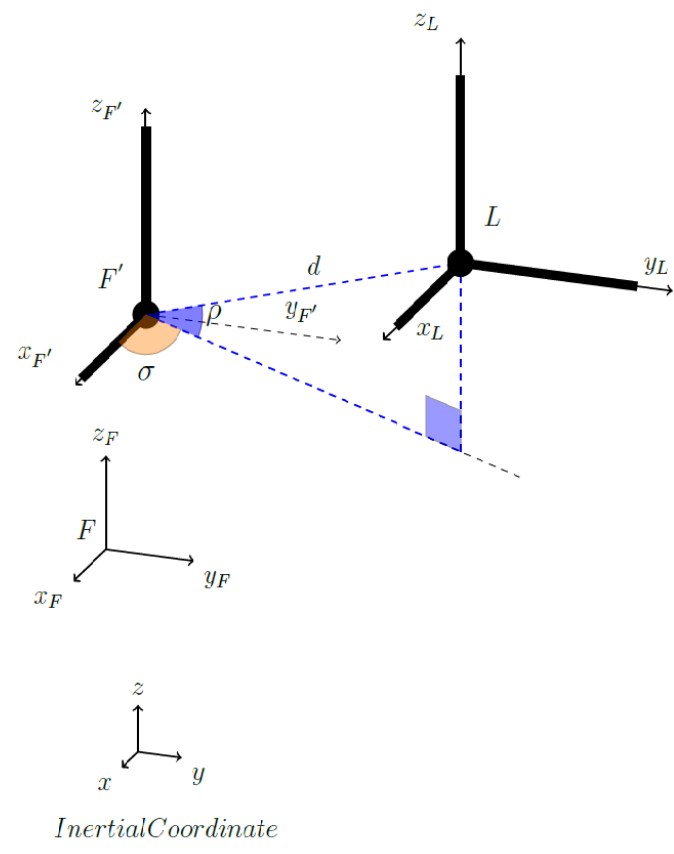

Fig. 1. Body Frames in Formation

$$
\left\{\begin{array}{l}
\lim _{t \rightarrow \infty}\left(\mathbf{p}_{L d}-\mathbf{p}_{L}\right)=0 \\
\lim _{t \rightarrow \infty}\left(\psi_{L d}-\psi_{L}\right)=0
\end{array}\right.
$$

In summary, the leader-follower formation control problem to be solved in this work is a distributed control scheme. Assume both the leader and the follower are able to obtain their own pose information and the follower is able to obtain the leader's pose information via wireless communication. The design goal of the controllers is to find the state feedback control law for the thrust and torque inputs for both the leader and the follower. The leader-follower formation control problem is solved if both conditions (2) and (3) are satisfied.

The communication among the robots is assumed to be available. The position $\mathbf{p}_{L}$, yaw angle $\psi_{L}$ of the leader $L$ and its first and second derivatives $\dot{\psi}_{L}$ and $\ddot{\psi}_{L}$ are assumed to be available and measurable. The linear velocity of the leader $L$ and its derivatives $\mathbf{v}_{L}$ and $\dot{\mathbf{v}}_{L}$ are assumed bounded and available for the follower.

\section{FORMATION $H_{\infty}$ CONTROLLERS}

The controller design for the leader and the follower is based on $H_{\infty}$ suboptimal control. The follower $H_{\infty}$ controller is designed by following the introduction of an error state model, and the introduction of a $H_{\infty}$ control theorem for general affine systems. Then the leader $H_{\infty}$ controller is briefly presented later.

\section{A. Follower State Error Model}

The control strategy for the follower is to track the desired position $\mathbf{p}_{F d}$. The tracking errors for the follower according to the nonlinear dynamic system (1) can be written as:

$$
\begin{aligned}
\tilde{\mathbf{p}}_{F} & =\mathbf{p}_{F d}-\mathbf{p}_{F} \\
\tilde{\mathbf{v}}_{F} & =\mathbf{v}_{F d}-\mathbf{v}_{F} \\
\tilde{\zeta}_{F} & =\zeta_{F d}-\zeta_{F} \\
\tilde{\eta}_{F} & =\eta_{F d}-\eta_{F}
\end{aligned}
$$

where $\mathbf{v}_{F d}=\dot{\mathbf{p}}_{F d}$ is the desired linear velocity, $\zeta_{F d}=$ $\left[0,0, \psi_{L}\right]^{T}$ is the desired angles, and $\eta_{F d}=[0,0,0]^{T}$ is the desired angles derivative. Then equation (1) can be rewritten in error form as:

$$
\left\{\begin{array}{l}
\dot{\tilde{\mathbf{p}}}_{F}=\tilde{\mathbf{v}}_{F} \\
\dot{\tilde{\mathbf{v}}}_{F}=\dot{\mathbf{v}}_{F d}+g \mathbf{e}-\frac{f_{F}}{m_{F}} R_{F} \mathbf{e} \\
\dot{\tilde{\zeta}}_{F}=\dot{\eta}_{F} \\
J_{F} \dot{\tilde{\eta}}_{F}=S\left(\tilde{\eta}_{F}\right) J_{F} \tilde{\eta}_{F}+G\left(\tilde{\eta}_{F}\right)-\tau_{F}
\end{array} .\right.
$$

Consider the external disturbances $\mathbf{d}_{F}=\left[\mathbf{d}_{\mathbf{v} F}^{T}, \mathbf{d}_{\eta F}^{T}\right]^{T}$ applied to the nonlinear system (4), where $\mathbf{d}_{\mathbf{v}_{F}}=\left[d_{v_{F x}}, d_{v_{F y}}, d_{v_{F z}}\right]^{T}$, $\mathbf{d}_{\eta_{F}}=\left[d_{F \dot{\varphi}}, d_{F \dot{\theta}}, d_{F \dot{\psi}}\right]^{T}$ are the disturbance vectors applied to $\tilde{\mathbf{p}}_{F}$ and $\tilde{\eta}_{F}$, respectively. Those disturbances are used here to model the changes of mass and moment, and the wind disturbances.

Let

$$
\begin{aligned}
\mathbf{x}_{F}=\left[\begin{array}{c}
\tilde{\mathbf{p}}_{F} \\
\tilde{\zeta}_{F} \\
\tilde{\mathbf{v}}_{F} \\
\tilde{\eta}_{F}
\end{array}\right] \\
\mathbf{u}_{F}=\left[\begin{array}{c}
\dot{\mathbf{v}}_{F d}+g \mathbf{e}-\frac{f_{F}}{m_{F}} R_{F} \mathbf{e} \\
G\left(\tilde{\eta}_{F}\right)-\tau_{F}
\end{array}\right] .
\end{aligned}
$$

The nonlinear dynamic system (4) with the disturbance vector $\mathbf{d}_{F}$ can be written into an affine nonlinear form:

$$
\dot{\mathbf{x}}_{F}=f\left(\mathbf{x}_{F}\right)+g\left(\mathbf{x}_{F}\right) \mathbf{u}_{F}+k\left(\mathbf{x}_{F}\right) \mathbf{d}_{F}
$$

where

$$
\begin{gathered}
f\left(\mathbf{x}_{F}\right)=\left[\begin{array}{c}
\tilde{\mathbf{v}}_{F} \\
\tilde{\eta}_{F} \\
0_{3 \times 1} \\
J_{F}^{-1} S\left(\tilde{\eta}_{F}\right) J_{F} \tilde{\eta}_{F}
\end{array}\right] \\
g\left(\mathbf{x}_{F}\right)=k\left(\mathbf{x}_{F}\right)=\left[\begin{array}{cc}
0_{3 \times 3} & 0_{3 \times 3} \\
0_{3 \times 3} & 0_{3 \times 3} \\
I & 0_{3 \times 3} \\
0_{3 \times 3} & J_{F}^{-1}
\end{array}\right] .
\end{gathered}
$$

\section{B. $H_{\infty}$ Suboptimal Control Approach}

In this section, an overview on the $H_{\infty}$ suboptimal control approach is summarised for affine nonlinear systems of the form:

$$
\begin{aligned}
& \dot{\mathbf{x}}=f(\mathbf{x})+g(\mathbf{x}) \mathbf{u}+k(\mathbf{x}) \mathbf{d} \\
& \mathbf{y}=h(\mathbf{x})
\end{aligned}
$$

where $\mathbf{x} \in \mathbb{R}^{n}$ is a state vector, $\mathbf{u} \in \mathbb{R}^{m}$ is an input vector, $\mathbf{y} \in \mathbb{R}^{p}$ is an output vector, and $\mathbf{d} \in \mathbb{R}^{q}$ is a disturbance vector. Detailed information on $H_{\infty}$ control approach can be found in [25]. 
We assume the existence of an equilibrium $\mathbf{x}_{*}$, i.e. $f\left(\mathbf{x}_{*}\right)=$ 0 , and we also assume $h\left(\mathbf{x}_{*}\right)=0$. Given a smooth state feedback controller,

$$
\left\{\begin{array}{l}
\mathbf{u}=l(\mathbf{x}) \\
l\left(\mathbf{x}_{*}\right)=0 .
\end{array}\right.
$$

The $H_{\infty}$ suboptimal control problem considers the $L_{2}$-gain from the disturbance $\mathbf{d}$ to the vector of $\mathbf{z}=\left[\mathbf{y}^{T}, \mathbf{u}^{T}\right]^{T}$. This problem is defined below.

Problem 1: Let $\gamma$ be a fixed nonnegative constant. The closed loop system consisting of the nonlinear system (6) and the state feedback controller (7) is said to have $L_{2}$-gain less than or equal to $\gamma$ from $\mathbf{d}$ to $\mathbf{z}$ if

$$
\int_{0}^{T}\|\mathbf{z}(t)\|^{2} d t \leq \gamma^{2} \int_{0}^{T}\|\mathbf{d}(t)\|^{2} d t+K(\mathbf{x}(0))
$$

for all $T \geq 0$ and all $\mathbf{d} \in L_{2}(0, T)$ with initial condition $\mathbf{x}(0)$, where $0 \leq K(\mathbf{x})<\infty$ and $K\left(\mathbf{x}_{*}\right)=0$.

For the nonlinear system (6) and $\gamma>0$, define the Hamiltonian $H_{\gamma}(\mathbf{x}, V(\mathbf{x}))$ as below:

$$
\begin{aligned}
H_{\gamma}(\mathrm{x}, V(\mathrm{x}))= & \frac{\partial V(\mathrm{x})}{\partial \mathrm{x}} f(\mathrm{x})+\frac{1}{2} \frac{\partial V(\mathrm{x})}{\partial \mathrm{x}} \\
& {\left[\frac{1}{\gamma^{2}} k(\mathrm{x}) k^{T}(\mathrm{x})-g(\mathrm{x}) g^{T}(\mathrm{x})\right] } \\
& \frac{\partial^{T} V(\mathrm{x})}{\partial \mathrm{x}}+\frac{1}{2} h^{T}(\mathrm{x}) h(\mathrm{x})
\end{aligned}
$$

Theorem 1: [25] If there exists a smooth solution $V \geq 0$ to the Hamilton-Jacobi inequality

$$
\begin{aligned}
H_{\gamma}(\mathbf{x}, V(\mathbf{x})) & \leq 0 \\
V\left(\mathbf{x}_{*}\right) & =0,
\end{aligned}
$$

then the closed-loop system for the state feedback controller

$$
\mathbf{u}=-g^{T}(\mathbf{x}) \frac{\partial^{T} V(\mathbf{x})}{\partial \mathbf{x}}
$$

has $L_{2}$-gain less than or equal to $\gamma$, and $K(\mathbf{x})=2 V(\mathbf{x})$.

The nonlinear system (6) is called zero-state observable if for any trajectory $\mathbf{x}(t)$ such that $\mathbf{y}(t)=0, \mathbf{u}(t)=0, \mathbf{d}(t)=0$ implies $\mathbf{x}(t)=\mathbf{x}_{*}$.

Proposition 1: [25] If the nonlinear system (6) is zerostate observable and there exists a proper solution $V \geq 0$ to the Hamilton-Jacobi inequality, then $V(\mathbf{x})>0$ for $\mathbf{x}(t) \neq \mathbf{x}_{*}$ and the closed loop system (6), (11) with $\mathbf{d}=0$ is globally asymptotically stable.

\section{Follower $H_{\infty}$ controller}

The $H_{\infty}$ suboptimal control approach will be used to design the follower controller in this section. The following form of energy function $V$ is suggested for the dynamic model (5):

$$
\begin{aligned}
V\left(\mathbf{x}_{F}\right)= & \frac{1}{2}\left[\begin{array}{llll}
\tilde{\mathbf{p}}_{F}^{T} & \tilde{\zeta}_{F}^{T} & \tilde{\mathbf{v}}_{F}^{T} & \tilde{\eta}_{F}^{T}
\end{array}\right] \\
& {\left[\begin{array}{cccc}
C_{F p} I & 0_{3 \times 3} & K_{F p} & 0_{3 \times 3} \\
0_{3 \times 3} & C_{F \zeta} I & 0_{3 \times 3} & J_{F} K_{F \zeta} \\
K_{F p} & 0_{3 \times 3} & K_{F v} & 0_{3 \times 3} \\
0_{3 \times 3} & J_{F} K_{F \zeta} & 0_{3 \times 3} & J_{F} K_{F \eta}
\end{array}\right]\left[\begin{array}{c}
\tilde{\mathbf{p}}_{F} \\
\tilde{\zeta}_{F} \\
\tilde{\mathbf{v}}_{F} \\
\tilde{\eta}_{F}
\end{array}\right] }
\end{aligned}
$$

where diagonal matrices $K_{F p}>0, K_{F \zeta}>0, K_{F v}>$ $0, K_{F \eta}>0$ are the proportional and derivative gains for translational and rotational parts. $C_{F p}>0, C_{F \zeta}>0$ are constants. We have:

$$
\begin{aligned}
& \frac{\partial V\left(\mathbf{x}_{F}\right)}{\partial \mathbf{x}_{F}}=\left[C_{F p} \tilde{\mathbf{p}}_{F}+K_{F p} \tilde{\mathbf{v}}_{F} \quad C_{F \zeta} \tilde{\zeta}_{F}+J_{F} K_{F \zeta} \tilde{\eta}_{F}\right. \\
& \left.K_{F p} \tilde{\mathbf{p}}_{F}+K_{F v} \tilde{\mathbf{v}}_{F} \quad J_{F} K_{F \zeta} \tilde{\zeta}_{F}+J_{F} K_{F \eta} \tilde{\eta}_{F}\right]
\end{aligned}
$$

Accordingly the controller is

$$
\begin{aligned}
\mathbf{u}_{F} & =-g^{T}\left(\mathbf{x}_{F}\right) \frac{\partial^{T} V\left(\mathbf{x}_{F}\right)}{\partial \mathbf{x}_{F}} \\
& =-\left[\begin{array}{c}
K_{F p} \tilde{\mathbf{p}}_{F}+K_{F v} \tilde{\mathbf{v}}_{F} \\
K_{F \zeta} \tilde{\zeta}_{F}+K_{F \eta} \tilde{\eta}_{F}
\end{array}\right]
\end{aligned}
$$

The following diagonal weighting matrices are chosen $W_{F 1}>0, W_{F 2}>0, W_{F 3}>0$ and $W_{F 4}>0$.

$h\left(\mathbf{x}_{F}\right)=\left[\begin{array}{llll}\sqrt{W_{F 1}} \cdot \tilde{\mathbf{p}}_{F}^{T} & \sqrt{W_{F 2}} \cdot \tilde{\zeta}_{F}^{T} & \sqrt{W_{F 3}} \cdot \tilde{\mathbf{v}}_{F}^{T} & \sqrt{W_{F 4}} \cdot \tilde{\eta}_{F}^{T}\end{array}\right]^{T}$ which satisfies $h\left(\mathbf{x}_{* F}\right)=0$, where the equilibrium point $\mathbf{x}_{* F}=\left[0_{1 \times 3}, 0_{1 \times 3}, 0_{1 \times 3}, 0_{1 \times 3}\right]^{T}$. And we know

$$
V\left(\mathbf{x}_{* F}\right)=0
$$

Now the team formation problem of the quadrotors under the disturbance $\mathbf{d}_{F}$ is defined below.

Problem 2: Given the equilibrium point $\mathbf{x}_{* F}$, find the parameters $K_{F p}, K_{F \zeta}, K_{F v}, K_{F \eta}, C_{F p}, C_{F \zeta}$ in order to enable the closed-loop system (5) with the above controller $\mathbf{u}_{F}$ (13) to have $L_{2}$-gain less than or equal to $\gamma_{F}$.

Next, our main result is represented in the following theorem.

Theorem 2: If the following conditions are satisfied, the closed-loop system (5) with the above controller $\mathbf{u}_{F}$ (13) has $L_{2}$-gain less than or equal to $\gamma_{F}$. And the closed loop system (5), (13) with $\mathbf{d}_{F}=0$ is asymptotically locally stable for the equilibrium point $\mathbf{x}_{* F}$.

$$
\begin{aligned}
C_{F p} C_{F \zeta} & \geq 0 \\
C_{F p} K_{F v} & \geq K_{F p}^{2} \\
C_{F p} C_{F \zeta} K_{F v} K_{F \eta} & \geq C_{F p} J_{F} K_{F \zeta}^{2} K_{F v}-J_{F} K_{F \zeta}^{2} K_{F p}^{2} \\
& +C_{F \zeta} K_{F p}^{2} K_{F \eta} \\
C_{F p} & =K_{F p} K_{F v}\left(1-\frac{1}{\gamma_{F}^{2}}\right) \\
C_{F \zeta} & =K_{F \zeta} K_{F \eta}\left(1-\frac{1}{\gamma_{F}^{2}}\right) \\
\left\|K_{F p}\right\|^{2} & \geq \frac{\gamma_{F}^{2}\left\|W_{F 1}\right\|}{\gamma_{F}^{2}-1} \\
\left\|K_{F \zeta}\right\|^{2} & \geq \frac{\gamma_{F}^{2}\left\|W_{F 2}\right\|}{\gamma_{F}^{2}-1} \\
\left\|K_{F v}\right\|^{2} & \geq \frac{\gamma_{F}^{2}\left(\left\|W_{F 3}\right\|+2\left\|K_{F p}\right\|\right)}{\gamma_{F}^{2}-1} \\
\left\|K_{F \eta}\right\|^{2} & \geq \frac{\gamma_{F}^{2}\left(\left\|W_{F 4}\right\|+2\left\|J_{F}\right\|\left\|K_{F \zeta}\right\|\right)}{\gamma_{F}^{2}-1} \\
\left\|W_{F 1}\right\| & >0 ;\left\|W_{F 2}\right\|>0 ;\left\|W_{F 3}\right\|>0 ;\left\|W_{F 4}\right\|>0 .
\end{aligned}
$$

Proof: With the given conditions, we need to show (1) $V(\mathbf{x}) \geq 0$ and (2) the Hamiltonian $H_{\gamma}(\mathbf{x}, V(\mathbf{x})) \leq 0$. Then 
the first part of the theorem can be proved by using Theorem 1. (1) From Equation (12) the conditions for $V(\mathbf{x}) \geq 0$ are

$$
\begin{aligned}
C_{F p} C_{F \zeta} & \geq 0 \\
C_{F p} K_{F v} & \geq K_{F p}^{2} \\
C_{F p} C_{F \zeta} K_{F v} K_{F \eta} & \geq C_{F p} J_{F} K_{F \zeta}^{2} K_{F v}-J_{F} K_{F \zeta}^{2} K_{F p}^{2} \\
& +C_{F \zeta} K_{F p}^{2} K_{F \eta}
\end{aligned}
$$

$$
\begin{aligned}
H_{\gamma_{F}}\left(\mathbf{x}_{F}, V\left(\mathbf{x}_{F}\right)\right) & =\tilde{\mathbf{p}}_{F}^{T} C_{F p} \tilde{\mathbf{v}}_{F}+\tilde{\mathbf{v}}_{F}^{T} K_{F p} \tilde{\mathbf{v}}_{F}+\tilde{\zeta}_{F}^{T} C_{F \zeta} \tilde{\eta}_{F} \\
& +\tilde{\eta}_{F}^{T} J_{F} K_{F \zeta} \tilde{\eta}_{F}+\tilde{\zeta}_{F}^{T} J_{F} K_{F \zeta} S\left(\tilde{\eta}_{F}\right) \tilde{\eta}_{F} \\
& +\tilde{\eta}_{F}^{T} J_{F} K_{F \eta} S\left(\tilde{\eta}_{F}\right) \tilde{\eta}_{F}+\frac{1}{2}\left(\frac{1}{\gamma_{F}^{2}}-1\right) \\
& \left\|K_{F p} \tilde{\mathbf{p}}_{F}+K_{F v} \tilde{\mathbf{v}}_{F}\right\|^{2}+\frac{1}{2}\left(\frac{1}{\gamma_{F}^{2}}-1\right) \\
& \left\|K_{F \zeta} \tilde{\zeta}_{F}+K_{F \eta} \tilde{\eta}_{F}\right\|^{2}+\frac{1}{2}\left\|W_{F 1}\right\|\left\|\tilde{\mathbf{p}}_{F}\right\|^{2} \\
& +\frac{1}{2}\left\|W_{F 2}\right\|\left\|\tilde{\zeta}_{F}\right\|^{2}+\frac{1}{2}\left\|W_{F 3}\right\|\left\|\tilde{\mathbf{v}}_{F}\right\|^{2} \\
& +\frac{1}{2}\left\|W_{F 4}\right\|\left\|\tilde{\eta}_{F}\right\|^{2} .
\end{aligned}
$$

By choosing

$$
\begin{aligned}
& C_{F p}=K_{F p} K_{F v}\left(1-\frac{1}{\gamma_{F}^{2}}\right) \\
& C_{F \zeta}=K_{F \zeta} K_{F \eta}\left(1-\frac{1}{\gamma_{F}^{2}}\right),
\end{aligned}
$$

then

$$
\begin{aligned}
H_{\gamma_{F}}\left(\mathbf{x}_{F}, V\left(\mathbf{x}_{F}\right)\right) & =\tilde{\mathbf{v}}_{F}^{T} K_{F p} \tilde{\mathbf{v}}_{F}+\tilde{\zeta}_{F}^{T} J_{F} K_{F \zeta} S\left(\tilde{\eta}_{F}\right) \tilde{\eta}_{F} \\
& +\tilde{\eta}_{F}^{T} J_{F} K_{F \zeta} \tilde{\eta}_{F}+\tilde{\eta}_{F}^{T} J_{F} K_{F \eta} S\left(\tilde{\eta}_{F}\right) \tilde{\eta}_{F} \\
& +\frac{1}{2}\left(\frac{1}{\gamma_{F}^{2}}-1\right)\left(\left\|K_{F p}\right\|^{2}\left\|\tilde{\mathbf{p}}_{F}\right\|^{2}\right. \\
& \left.+\left\|K_{F v}\right\|^{2}\left\|\tilde{\mathbf{v}}_{F}\right\|^{2}\right)+\frac{1}{2}\left(\frac{1}{\gamma_{F}^{2}}-1\right) \\
& \left(\left\|K_{F \zeta}\right\|^{2}\left\|\tilde{\zeta}_{F}\right\|^{2}+\left\|K_{F \eta}\right\|^{2}\left\|\tilde{\eta}_{F}\right\|^{2}\right) \\
& +\frac{1}{2}\left\|W_{F 1}\right\|\left\|\tilde{\mathbf{p}}_{F}\right\|^{2}+\frac{1}{2}\left\|W_{F 2}\right\|\left\|\tilde{\zeta}_{F}\right\|^{2} \\
& +\frac{1}{2}\left\|W_{F 3}\right\|\left\|\tilde{\mathbf{v}}_{F}\right\|^{2}+\frac{1}{2}\left\|W_{F 4}\right\|\left\|\tilde{\eta}_{F}\right\|^{2} .
\end{aligned}
$$

By using $\left|\tilde{\mathbf{v}}_{F}^{T} K_{F p} \tilde{\mathbf{v}}_{F}\right| \leq\left\|K_{F p}\right\|\left\|\tilde{\mathbf{v}}_{F}\right\|^{2},\left\|S\left(\tilde{\eta}_{F}\right)\right\|=$ $\left\|\tilde{\eta}_{F}\right\|, \quad\left\|\tilde{\eta}_{F}^{T} J_{F} K_{F \eta} S\left(\tilde{\eta}_{F}\right) \tilde{\eta}_{F}\right\|=0, \quad\left|\tilde{\eta}_{F}^{T} J_{F} K_{F \zeta} \tilde{\eta}_{F}\right| \leq$ $\left\|J_{F}\right\|\left\|K_{F \zeta}\right\|\left\|\tilde{\eta}_{F}\right\|^{2}$ and $\left|\tilde{\zeta}_{F}^{T} J_{F} K_{F \eta} S\left(\tilde{\eta}_{F}\right) \tilde{\eta}_{F}\right|=0$, we have

$$
\begin{aligned}
H_{\gamma_{F}}\left(\mathbf{x}_{F}, V\left(\mathbf{x}_{F}\right)\right) & =\left\|K_{F p}\right\|\left\|\tilde{\mathbf{v}}_{F}\right\|^{2}+\left\|J_{F}\right\|\left\|K_{F \zeta}\right\|\left\|\tilde{\eta}_{F}\right\|^{2} \\
& +\frac{1}{2}\left(\frac{1}{\gamma_{F}^{2}}-1\right)\left(\left\|K_{F p}\right\|^{2}\left\|\tilde{\mathbf{p}}_{F}\right\|^{2}\right. \\
& \left.+\left\|K_{F v}\right\|^{2}\left\|\tilde{\mathbf{v}}_{F}\right\|^{2}\right)+\frac{1}{2}\left(\frac{1}{\gamma_{F}^{2}}-1\right) \\
& \left(\left\|K_{F \zeta}\right\|^{2}\left\|\tilde{\zeta}_{F}\right\|^{2}+\left\|K_{F \eta}\right\|^{2}\left\|\tilde{\eta}_{F}\right\|^{2}\right) \\
& +\frac{1}{2}\left\|W_{F 1}\right\|\left\|\tilde{\mathbf{p}}_{F}\right\|^{2}+\frac{1}{2}\left\|W_{F 2}\right\|\left\|\tilde{\zeta}_{F}\right\|^{2} \\
& +\frac{1}{2}\left\|W_{F 3}\right\|\left\|\tilde{\mathbf{v}}_{F}\right\|^{2}+\frac{1}{2}\left\|W_{F 4}\right\|\left\|\tilde{\eta}_{F}\right\|^{2} .
\end{aligned}
$$

Thus, the conditions for $H_{\gamma_{F}}\left(\mathbf{x}_{F}, V\left(\mathbf{x}_{F}\right)\right) \leq 0$ are

$$
\begin{aligned}
& \frac{1}{2}\left(\frac{1}{\gamma_{F}^{2}}-1\right)\left\|K_{F p}\right\|^{2}+\frac{1}{2}\left\|W_{F 1}\right\| \leq 0 \\
& \frac{1}{2}\left(\frac{1}{\gamma_{F}^{2}}-1\right)\left\|K_{F \zeta}\right\|^{2}+\frac{1}{2}\left\|W_{F 2}\right\| \leq 0 \\
\left\|K_{F p}\right\|+ & \frac{1}{2}\left(\frac{1}{\gamma_{F}^{2}}-1\right)\left\|K_{F v}\right\|^{2}+\frac{1}{2}\left\|W_{F 3}\right\| \leq 0 \\
\left\|J_{F}\right\|\left\|K_{F \zeta}\right\|+ & \frac{1}{2}\left(\frac{1}{\gamma_{F}^{2}}-1\right)\left\|K_{F \eta}\right\|^{2}+\frac{1}{2}\left\|W_{F 4}\right\| \leq 0 ;
\end{aligned}
$$

i.e.

$$
\begin{aligned}
\left\|K_{F p}\right\|^{2} & \geq \frac{\gamma_{F}^{2}\left\|W_{F 1}\right\|}{\gamma_{F}^{2}-1} \\
\left\|K_{F \zeta}\right\|^{2} & \geq \frac{\gamma_{F}^{2}\left\|W_{F 2}\right\|}{\gamma_{F}^{2}-1} \\
\left\|K_{F v}\right\|^{2} & \geq \frac{\gamma_{F}^{2}\left(\left\|W_{F 3}\right\|+2\left\|K_{F p}\right\|\right)}{\gamma_{F}^{2}-1} \\
\left\|K_{F \eta}\right\|^{2} & \geq \frac{\gamma_{F}^{2}\left(\left\|W_{F 4}\right\|+2\left\|J_{F}\right\|\left\|K_{F \zeta}\right\|\right)}{\gamma_{F}^{2}-1} .
\end{aligned}
$$

It is trivial to show that the nonlinear system (5) is zero-state observable for the equilibrium point $\mathbf{x}_{* F}$. Further, due to the fact that $V\left(\mathbf{x}_{F}\right) \geq 0$ and it is a proper function (i.e. for each $\beta>0$ the set $\left\{x_{F}: 0 \leq V\left(x_{F}\right) \leq \beta\right\}$ is compact), the closedloop system (5), (13) with $\mathbf{d}_{F}=0$ is asymptotically locally stable for the equilibrium point $\mathbf{x}_{* F}$ according to Proposition 1. This proves the second part of the theorem.

Remark 1: It should be noted that the proof of Theorem 2, $\lim _{t \rightarrow \infty} \tilde{\mathbf{p}}=0, \lim _{t \rightarrow \infty} \tilde{\zeta}=0, \lim _{t \rightarrow \infty} \tilde{\mathbf{v}}=0$ and $\lim _{t \rightarrow \infty} \tilde{\eta}=0$ meets the conditions of (2).

Then from $\mathbf{u}_{F}$, we can have

$$
\begin{aligned}
\mathbf{u}_{F} & =\left[\begin{array}{c}
\dot{\mathbf{v}}_{F d}+g \mathbf{e}-\frac{f_{F}}{m_{F}} R_{F} \mathbf{e} \\
G\left(\tilde{\eta}_{F}\right)-\tau_{F}
\end{array}\right] \\
& =-\left[\begin{array}{c}
K_{F p} \tilde{\mathbf{p}}_{F}+K_{F v} \tilde{\mathbf{v}}_{F} \\
K_{F \zeta} \tilde{\zeta}_{F}+K_{F \eta} \tilde{\eta}_{F}
\end{array}\right] .
\end{aligned}
$$

Then the total force and the torque vector are applied to the follower, $f_{F}$ and $\tau_{F} \in \mathbb{R}^{3}$;

$$
\begin{aligned}
f_{F}= & \left(k_{F z} \tilde{z}_{F}+k_{F v_{z}} \tilde{v}_{F z}+\dot{v}_{L z}-d\left(R_{31} \cos \rho \cos \sigma\right.\right. \\
& \left.\left.+R_{32} \cos \rho \sin \sigma+R_{33} \sin \rho\right)+g\right) \frac{m_{F}}{c \varphi_{L} c \theta_{L}} \\
\tau_{F}= & K_{F \zeta} \tilde{\zeta}_{F}+K_{F \eta} \tilde{\eta}_{F}+G\left(\tilde{\eta}_{F}\right)
\end{aligned}
$$

where

$$
\ddot{R}_{L}^{T}=\left[\begin{array}{ccc}
R_{11} & R_{12} & R_{13} \\
R_{21} & R_{22} & R_{23} \\
R_{31} & R_{32} & R_{33}
\end{array}\right] .
$$

\section{Leader $H_{\infty}$ controller}

The control strategy for the leader is to track a desired trajectory $\left(\mathbf{p}_{L d}, \psi_{L d}\right)$. The tracking errors for the leader according to the nonlinear dynamic system (1) can be written 
as:

$$
\begin{aligned}
\tilde{\mathbf{p}}_{L} & =\mathbf{p}_{L d}-\mathbf{p}_{L} \\
\tilde{\mathbf{v}}_{L} & =\mathbf{v}_{L d}-\mathbf{v}_{L} \\
\tilde{\eta}_{L} & =\eta_{L d}-\eta_{L} \\
\tilde{\zeta}_{L} & =\zeta_{L d}-\zeta_{L}
\end{aligned}
$$

where $\eta_{L d}, \mathbf{v}_{L d}, \zeta_{L d}$ are assumed to be constant for the desired tracking trajectory. Then equation (1) can be rewritten in an error form as:

$$
\left\{\begin{array}{l}
\dot{\tilde{\mathbf{p}}}_{L}=\tilde{\mathbf{v}}_{L} \\
\dot{\tilde{\mathbf{v}}}_{L}=g \mathbf{e}-\frac{f_{L}}{m_{L}} R_{L} \mathbf{e} \\
\dot{\tilde{\zeta}}_{L}=\tilde{\eta}_{L} \\
J_{L} \dot{\tilde{\eta}}_{L}=S\left(\tilde{\eta}_{L}\right) J_{L} \tilde{\eta}_{L}+G\left(\tilde{\eta}_{L}\right)-\tau_{L}
\end{array} .\right.
$$

Let

$$
\begin{aligned}
\mathbf{x}_{L}= & {\left[\begin{array}{c}
\tilde{\mathbf{p}}_{L} \\
\tilde{\zeta}_{L} \\
\tilde{\mathbf{v}}_{L} \\
\tilde{\eta}_{L}
\end{array}\right] } \\
\mathbf{u}_{L}= & {\left[\begin{array}{c}
g \mathbf{e}-\frac{f_{L}}{m_{L}} R_{L} \mathbf{e} \\
G\left(\tilde{\eta}_{L}\right)-\tau_{L}
\end{array}\right] . }
\end{aligned}
$$

The nonlinear dynamic system (19) with the disturbance vector $\mathbf{d}_{L}$ can be written into an affine nonlinear form:

$$
\dot{\mathbf{x}}_{L}=f\left(\mathbf{x}_{L}\right)+g\left(\mathbf{x}_{L}\right) \mathbf{u}_{L}+k\left(\mathbf{x}_{L}\right) \mathbf{d}_{L}
$$

where

$$
\begin{gathered}
f\left(\mathbf{x}_{L}\right)=\left[\begin{array}{c}
\tilde{\mathbf{v}}_{L} \\
\tilde{\eta}_{L} \\
0_{3 \times 1} \\
J_{L}^{-1} S\left(\tilde{\eta}_{L}\right) J_{L} \tilde{\eta}_{L}
\end{array}\right] \\
g\left(\mathbf{x}_{L}\right)=k\left(\mathbf{x}_{L}\right)=\left[\begin{array}{cc}
0_{3 \times 3} & 0_{3 \times 3} \\
0_{3 \times 3} & 0_{3 \times 3} \\
I & 0_{3 \times 3} \\
0_{3 \times 3} & J_{L}^{-1}
\end{array}\right] .
\end{gathered}
$$

The $H_{\infty}$ suboptimal control approach is used to design the leader controller. By defining an energy function, the leader controller is obtained as below by following a similar procedure for stability analysis.

$$
\left\{\begin{array}{l}
f_{L}=\left(k_{L z} \tilde{z}_{L}+k_{L v_{z}} \tilde{v}_{L z}+g\right) \frac{m_{L}}{c \varphi_{L} c \theta_{L}} \\
\tau_{L}=K_{L \zeta} \tilde{\zeta}_{L}+K_{L \eta} \tilde{\eta}_{L}+G\left(\tilde{\eta}_{L}\right)
\end{array}\right.
$$

where $\tilde{\mathbf{p}}_{L}=\left[\tilde{x}_{L}, \tilde{y}_{L}, \tilde{z}_{L}\right]^{T}$ is the position tracking error vector and $\tilde{\mathbf{v}}_{L}=\left[\tilde{v}_{L x}, \tilde{v}_{L y}, \tilde{v}_{L z}\right]^{T}$ is the linear velocity error vector. The diagonal matrices $K_{L p}=\operatorname{diag}\left(k_{L x}, k_{L y}, k_{L z}\right)$, $K_{L v}=\operatorname{diag}\left(k_{L v_{x}}, k_{L v_{y}}, k_{L v_{z}}\right), K_{L \zeta}=\operatorname{diag}\left[k_{L \varphi}, k_{L \theta}, k_{L \psi}\right]$, $K_{L \eta}=\operatorname{diag}\left(k_{L \dot{\varphi}}, k_{L \dot{\theta}}, k_{L \dot{\psi}}\right)$ are selected to satisfy the stability conditions, which have been presented in [26].

\section{INTEGRAL BACKSTEPPING FORMATION CONTROL}

Integral backstepping control is one of the popular control approaches for both individual and multiple quadrotors. In this section, the IBS controller is applied for the individual quadrotor path tracking and leader-follower formation problems. An IBS controller for the follower is developed first. The IBS control law for the leader is also presented in this section. Its main result is also used later in simulation for evaluating the robustness of $H_{\infty}$ controllers.

\section{A. Follower integral backstepping controller}

The IBS controller for the follower is to track the leader and maintain a desired distance between them with desired incidence and bearing angles. In this subsection, we derive the IBS controller for the follower. Let us recall the follower translational part (1):

$$
\ddot{\mathbf{p}}_{F}=f\left(\mathbf{p}_{F}\right)+g\left(\mathbf{p}_{F}\right) f_{F}
$$

where

$$
\begin{gathered}
f\left(\mathbf{p}_{F}\right)=\left[\begin{array}{lll}
0 & 0 & -g
\end{array}\right]^{T} \\
g\left(\mathbf{p}_{F}\right)=\left[\begin{array}{lll}
u_{F x} / m_{F} & u_{F y} / m_{F} & c \theta_{F} c \varphi_{F} / m_{F}
\end{array}\right]^{T}
\end{gathered}
$$

where

$$
\begin{aligned}
& u_{F x}=\left(c \psi_{F} s \theta_{F} c \varphi_{F}+s \psi_{F} s \varphi_{F}\right) \\
& u_{F y}=\left(s \psi_{F} s \theta_{F} c \varphi_{F}-c \psi_{F} s \varphi_{F}\right) .
\end{aligned}
$$

Then the position tracking error between the leader and the follower can be calculated as:

$$
\tilde{\mathbf{p}}_{F}=\mathbf{p}_{F d}-\mathbf{p}_{F}=\mathbf{p}_{L}-R_{L}^{T} d\left[\begin{array}{c}
\cos \rho \cos \sigma \\
\cos \rho \sin \sigma \\
\sin \rho
\end{array}\right]-\mathbf{p}_{F}
$$

and its derivative is

$$
\dot{\tilde{\mathbf{p}}}_{F}=\dot{\mathbf{p}}_{F d}-\dot{\mathbf{p}}_{F}=\dot{\mathbf{p}}_{F d}-\mathbf{v}_{F}
$$

where $\mathbf{v}_{F}$ is a virtual control, and its desirable value can be described as:

$$
\mathbf{v}_{F}^{d}=\dot{\mathbf{p}}_{F d}+b_{F} \tilde{\mathbf{p}}_{F}+k_{F} \overline{\mathbf{p}}_{F}
$$

where $b_{F}$ and $k_{F}$ are two positive matrices, $\overline{\mathbf{p}}_{F}=\int \tilde{\mathbf{p}}_{F} d t$ is the integral of the follower position error and it is added to minimize the steady-state error.

Now, consider the linear velocity error between the leader and the follower as:

$$
\tilde{\mathbf{v}}_{F}=\mathbf{v}_{F}^{d}-\dot{\mathbf{p}}_{F} .
$$

By substituting (24) into (25) we obtain

$$
\tilde{\mathbf{v}}_{F}=\dot{\mathbf{p}}_{F d}+b_{F} \tilde{\mathbf{p}}_{F}+k_{F} \overline{\mathbf{p}}_{F}-\dot{\mathbf{p}}_{F}
$$

and its time derivative becomes

$$
\dot{\tilde{\mathbf{v}}}_{F}=\ddot{\mathbf{p}}_{F d}+b_{F} \dot{\tilde{\mathbf{p}}}_{F}+k_{F} \tilde{\mathbf{p}}_{F}-\ddot{\mathbf{p}}_{F} .
$$


Then from (24) and (25) we can rewrite (23) in terms of the linear velocity error as:

$$
\dot{\tilde{\mathbf{p}}}_{F}=\tilde{\mathbf{v}}_{F}-b_{F} \tilde{\mathbf{p}}_{F}-k_{F} \overline{\mathbf{p}}_{F} .
$$

By substituting (21) and (28) into (27), the time derivative of the linear velocity error can be rewritten as:

$$
\begin{aligned}
\dot{\tilde{\mathbf{v}}}_{F}= & \ddot{\mathbf{p}}_{F d}+b_{F} \tilde{\mathbf{v}}_{F}-b_{F}^{2} \dot{\tilde{\mathbf{p}}}_{F}-b_{F} k_{F} \overline{\mathbf{p}}_{F}+k_{F} \tilde{\mathbf{p}}_{F} \\
& -f\left(\mathbf{p}_{F}\right)-g\left(\mathbf{p}_{F}\right) f_{F} .
\end{aligned}
$$

The desirable time derivative of the linear velocity error is supposed to be

$$
\dot{\tilde{\mathbf{v}}}_{F}=-c_{F} \tilde{\mathbf{v}}_{F}-\tilde{\mathbf{p}}_{F}
$$

where $c_{F}$ is a positive diagonal matrix. Now, the total thrust $f_{F}$, the longitudinal $u_{F x}$ and lateral $u_{F y}$ motion control can be found by subtracting (29) from (30) as follows:

$$
\begin{aligned}
f_{F}= & \left(g+\dot{v}_{L z}+\left(1-b_{F z}^{2}+k_{F z}\right) \tilde{z}_{F}+\left(b_{F z}+c_{F z}\right) \tilde{v}_{F z}\right. \\
& -b_{F z} k_{F z} \bar{z}_{F}-d\left(R_{31} \cos \rho \cos \sigma+R_{32} \cos \rho \sin \sigma\right. \\
& \left.\left.+R_{33} \sin \rho\right)\right) \frac{m_{F}}{c \theta_{F} c \varphi_{F}} \\
u_{F x}= & \left(\dot{v}_{L x}+\left(1-b_{F x}^{2}+k_{F x}\right) \tilde{x}_{F}+\left(b_{F x}+c_{F x}\right) \tilde{v}_{F x}\right. \\
& -b_{F x} k_{F x} \bar{x}_{F}-d\left(R_{11} \cos \rho \cos \sigma+R_{12} \cos \rho \sin \sigma\right. \\
& \left.\left.+R_{13} \sin \rho\right)\right) \frac{m_{F}}{f_{F}} \\
u_{F y}= & \dot{v}_{L y}+\left(1-b_{F y}^{2}+k_{F y}\right) \tilde{y}_{F}+\left(b_{F y}+c_{F y}\right) \tilde{v}_{F y} \\
& -b_{F y} k_{F y} \bar{y}_{F}-d\left(R_{21} \cos \rho \cos \sigma+R_{22} \cos \rho \sin \sigma\right. \\
& \left.\left.+R_{23} \sin \rho\right)\right) \frac{m_{F}}{f_{F}} .
\end{aligned}
$$

For the attitude stability, the following $H_{\infty}$ nonlinear controller is used:

$$
\tau_{F}=K_{F \zeta} \tilde{\zeta}_{F}+K_{F \eta} \tilde{\eta}_{F}+G\left(\tilde{\eta}_{F}\right) .
$$

The attitude stability for the follower was demonstrated in [26] . Next, we show the stability of follower's translational part.

\section{B. Follower Controller stability analysis}

The following candidate Lyapunov function is chosen for the stability analysis for the follower's translational part with the IBS controller:

$$
V=\frac{1}{2}\left(\tilde{\mathbf{p}}_{F}^{T} \tilde{\mathbf{p}}_{F}+\tilde{\mathbf{v}}_{F}^{T} \tilde{\mathbf{v}}_{F}+k_{F} \overline{\mathbf{p}}_{F}^{T} \overline{\mathbf{p}}_{F}\right)
$$

and its time derivative is

$$
\dot{V}=\tilde{\mathbf{p}}_{F}^{T} \dot{\tilde{\mathbf{p}}}_{F}+\tilde{\mathbf{v}}_{F}^{T} \dot{\tilde{\mathbf{v}}}_{F}+k_{F} \overline{\mathbf{p}}_{F}^{T} \dot{\overline{\mathbf{p}}}_{F} .
$$

By substituting $\dot{\overline{\mathbf{p}}}_{F}=\tilde{\mathbf{p}}_{F}$ and Equations (28) and (30) into (35), Equation (35) becomes

$$
\dot{V}=-b_{F} \tilde{\mathbf{p}}_{F}^{T} \tilde{\mathbf{p}}_{F}-c_{F} \tilde{\mathbf{v}}_{F}^{T} \tilde{\mathbf{v}}_{F} \leq 0 .
$$

Finally, (36) is less than zero provided $b_{F}$ and $c_{F}$ are positive diagonal matrices, i.e. $\dot{V}<0, \forall\left(\tilde{\mathbf{p}}_{F}, \tilde{\mathbf{v}}_{F}\right) \neq 0$ and $\dot{V}(0)=0$. It can be concluded from the positive definition of $V$ and applying LaSalle theorem that a global asymptotic stability is guaranteed. This leads us to conclude that $\lim _{t \rightarrow \infty} \tilde{\mathbf{p}}_{F}=0$ and $\lim _{t \rightarrow \infty} \tilde{\mathbf{v}}_{F}=0$, which meets the position condition of (2).

\section{Leader integral backstepping controller}

The leader is to track a desired trajectory $\mathbf{p}_{L d}$. Its IBS controller is developed by following the procedure described for the follower quadrotor. The result is that the total force and horizontal position control laws $f_{L}, u_{L x}$ and $u_{L y}$ can be written as:

$$
\begin{aligned}
f_{L}= & \left(\ddot{z}_{L d}+g+\left(1-b_{L z}{ }^{2}+k_{L z}\right) \tilde{z}_{L}+\left(b_{L z}+c_{L z}\right) \tilde{v}_{L z}\right. \\
& \left.-b_{L z} k_{L z} \bar{z}_{L}\right) \frac{m_{L}}{c \theta_{L} c \varphi_{L}} \\
u_{L x} & =\left(\ddot{x}_{L d}+\left(1-b_{L x}{ }^{2}+k_{L x}\right) \tilde{x}_{L}+\left(b_{L x}+c_{L x}\right) \tilde{v}_{L x}\right. \\
& \left.-b_{L x} k_{L x} \bar{x}_{L}\right) \frac{m_{L}}{f_{L}} \\
u_{L y} & =\left(\ddot{y}_{L d}+\left(1-b_{L y}{ }^{2}+k_{L y}\right) \tilde{y}_{L}+\left(b_{L y}+c_{L y}\right) \tilde{v}_{L y}\right. \\
& \left.-b_{L y} k_{L y} \bar{y}_{L}\right) \frac{m_{L}}{f_{L}}
\end{aligned}
$$

and the linear velocity tracking errors are defined as:

$$
\left\{\begin{array}{l}
\tilde{v}_{L x}=b_{L x} \tilde{x}_{L}+\dot{x}_{L d}+k_{L x} \bar{x}_{L}-\dot{x}_{L} \\
\tilde{v}_{L y}=b_{L y} \tilde{y}_{L}+\dot{y}_{L d}+k_{L y} \bar{y}_{L}-\dot{y}_{L} \\
\tilde{v}_{L z}=b_{L z} \tilde{z}_{L}+\dot{z}_{L d}+k_{L z} \bar{z}_{L}-\dot{z}_{L}
\end{array} .\right.
$$

The torque vector applied to the leader quadrotor $\tau_{L} \in \mathbb{R}^{3}$ is designed as:

$$
\tau_{L}=K_{L \zeta} \tilde{\zeta}_{L}+K_{L \eta} \tilde{\eta}_{L}+G\left(\tilde{\eta}_{L}\right)
$$

\section{CONTROLLERS COMPARISON}

To further verifying the proposed $H_{\infty}$ control technique, a comparison with integral backstepping controller for leaderfollower formation of quadrotors has introduced in this section. Its well-known that integral backstepping control is a methodical approach to build the Lyapunov function ahead with the control input design. Thus by cancellation of the indefinite error terms, the stability of the derivative of the Lyapunov function be secured. Although the stability of the Lyapunov function is guaranteed this does not guaranteed the performance of the system. While the $H_{\infty}$ control technique is to convert the control problem to mathematical optimization problem and solve this optimization problem by finding a suitable controller. $H_{\infty}$ controller achieves the stabilization along with performance guaranteed.

It can be noted that the adjusting of the $H_{\infty}$ controller parameters is easer than adjusting that of integral backstepping. The former has two insulated parameters and it calculated mathematically depends on the attenuation parameter value $\gamma$. In contrast, the latter has three coupling parameters to be tuned manually to find the suitable values which take longer time and high effort.

Simulation results prove that the performance of using $H_{\infty}$ controller has significantly smaller errors than that of using integral backstepping. It is also obvious that using $H_{\infty}$ controller leads to a smooth and fast performance with very small overshoot compared with that of using integral backstepping. Moreover, the respond of using $H_{\infty}$ controller to reject the external disturbances is faster than that of using 


\begin{tabular}{|c||c|c|c|}
\hline Symbol & Definition & Value & Units \\
\hline$J_{x}$ & Roll Inertia & $4.4 \times 10^{-} 3$ & $\mathrm{~kg} . \mathrm{m}^{2}$ \\
\hline$J_{y}$ & Pitch Inertia & $4.4 \times 10^{-} 3$ & $\mathrm{~kg} . \mathrm{m}^{2}$ \\
\hline$J_{z}$ & Yaw Inertia & $8.8 \times 10^{-} 3$ & $\mathrm{~kg} . \mathrm{m}^{2}$ \\
\hline$m$ & Mass & 0.5 & $\mathrm{~kg}$ \\
\hline$g$ & Gravity & 9.81 & $\mathrm{~m} / \mathrm{s}^{2}$ \\
\hline$l$ & Arm Length & 0.17 & $\mathrm{~m}$ \\
\hline$J_{r}$ & Rotor Inertia & $4.4 \times 10^{-} 5$ & $\mathrm{~kg} . \mathrm{m}^{2}$ \\
\hline
\end{tabular}

TABLE I

QUADROTOR PARAMETERS

integral backstepping. A scenario of two identical quadrotors using a MATLAB simulator was considered to track a desired path for the leader and maintain the desired distance, desired incidence angle and desired bearing angle between them for the follower. The quadrotor parameters used in the simulation are described in Table I.

Two paths were presented in the simulation to show the performance of using the proposed $H_{\infty}$ controller with four different circumstances. The first desired path to be tracked by the leader was

$$
\left\{\begin{array}{l}
x_{L d}=2 \cos (t \pi / 80) \\
z_{L d}=1+0.1 t \quad ; \quad \psi_{L d}=0
\end{array} .\right.
$$

The leader initial positions were $\left[x_{L}, y_{L}, z_{L}\right]^{T}=[2,0,0]^{T}$ metres and the initial angles were $\left[\varphi_{L}, \theta_{L}, \psi_{L}\right]^{T}=[0,0,0]^{T}$ radian. Then the follower followed the leader and maintained the desired distance between them $d=2$ metres, the desired incidence and bearing angles $\rho=-\pi / 6, \sigma=$ $\pi / 6$ radian, respectively. The follower initial positions were $\left[x_{F}, y_{F}, z_{F}\right]^{T}=[0.5,0,0]^{T}$ metres and the initial angles were $\left[\varphi_{F}, \theta_{F}, \psi_{F}\right]^{T}=[0,0,0]^{T}$ radian. The second desired path to be tracked by the leader was

$$
\left\{\begin{array}{l}
x_{L d}=4 \cos (t \pi / 40) \\
z_{L d}=1+0.1 t \quad ; \quad \psi_{L d}=\pi / 6
\end{array} .\right.
$$

The leader initial positions were $\left[x_{L}, y_{L}, z_{L}\right]^{T}=[4,0,0]^{T}$ metres and the initial angles were $\left[\varphi_{L}, \theta_{L}, \psi_{L}\right]^{T}=[0,0,0]^{T}$ radian. Then the follower followed the leader and maintained the desired distance between them $d=3$ metres, the desired incidence and bearing angles $\rho=0, \sigma=$ $\pi / 6$ radian, respectively. The follower initial positions were $\left[x_{F}, y_{F}, z_{F}\right]^{T}=[1.4,-1.5,0]^{T}$ metres and the initial angles were $\left[\varphi_{F}, \theta_{F}, \psi_{F}\right]^{T}=[0,0,0]^{T}$ radian.

Figures 2 and 5 indicate the response of the proposed $H_{\infty}$ and integral backstepping controllers when the leader tracked the first desired path, respectively. Figure ?? shows the distances between the leader and the follower via the two controllers, and Figures 3, 4, 6 and 7 illustrate the yaw angle behaviour for the leader and the follower via the two controllers, respectively. For the second path, Figures 8 and 11 show the proposed $H_{\infty}$ and integral backstepping controllers positions respectively, Figures 9, 10, 12 and 13 illustrate the yaw angle of the leader and the follower via the two controllers respectively, and Figure 14 shows the distances between the leader and the follower via the two controllers. Its four circumstances included: (1) no disturbance, (2) force disturbance $d_{v_{i x}}=-2 \mathrm{Nm}$ during $10 \leq t \geq 10.25$



Fig. 2. Leader-Follower Formation in First Path under $H_{\infty}$ Controller

seconds, $d_{v_{i z}}=2 \mathrm{Nm}$ during $20 \leq t \geq 20.25$ seconds, $d_{v_{i y}}=2 \mathrm{Nm}$ during $30 \leq t \geq 30.25$ seconds in the first path, $d_{v_{i x}}=-2 \mathrm{Nm}$ during $20 \leq t \geq 20.25$ seconds, $d_{v_{i z}}=2 \mathrm{Nm}$ during $60 \leq t \geq 60.25$ seconds, $d_{v_{i y}}=2 \mathrm{Nm}$ during $100 \leq t \geq 100.25$ seconds in the second path, and $d_{i \dot{\varphi}}=d_{i \dot{\theta}}=d_{i \dot{\psi}}=0.01+0.01 \sin (0.024 \pi t)+0.05 \sin (1.32 \pi t)$, applied at the same time for both the leader and the follower, (3) $+30 \%$ model parameter uncertainty, and (4) $-30 \%$ model parameter uncertainty.

Figures 16 and 17 show the performance of both paths when only the leader was affected by force disturbance $d_{v_{L x}}=-4 \mathrm{Nm}$ during $20 \leq t \geq 20.25$ seconds, $d_{v_{L z}}=4 \mathrm{Nm}$ during $60 \leq t \geq 60.25$ seconds, $d_{v_{L y}}=4 \mathrm{Nm}$ during $100 \leq$ $t \geq 100.25$ seconds, and the leader attitude part is disturbed using $d_{L \dot{\varphi}}=d_{L \dot{\theta}}=d_{L \dot{\psi}}=0.01+0.01 \sin (0.024 \pi t)+$ $0.05 \sin (1.32 \pi t)$.

From Figures $2-17$ the overshoots of using the $H_{\infty}$ controller were very small and the RMSE values of the desired distances between the leader and the follower were also very small and the controller's performance was fast in rejecting the disturbances as well compared with those of the IBS controller. Moreover, the IBS controller could not reject the disturbances in the second path. As a result, the proposed $H_{\infty}$ controller indeed produced excellent control performance.

\section{EXPERIMENTAL RESULTS}

Experimental results are presented in this section with one leader and one follower tested by using three different paths in an indoor flight environment with consideration of external disturbances and weight changes. The leader tracked a predefined path, then the follower used the leader's actual position to calculate its path to follow. The information used to control the leader and the follower was received from the IMU and the Vicon Motion Capture System. Figure 18 shows the control diagram used in this work; here it can be noticed that the communication between the computer and the quadrotors was linked via two Xbees: the first one was mounted on the quadrotor while the second one was connected to the computer. The position of the leader was sent to the computer, and then it was sent from the computer to the follower to be used for 


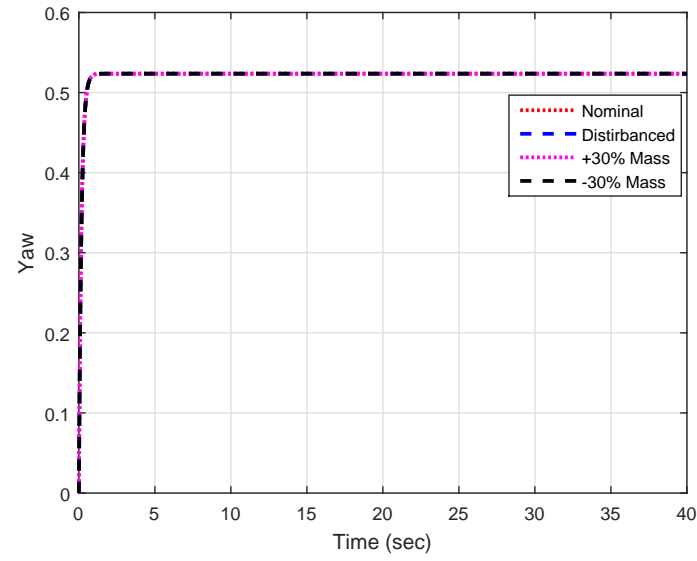

Fig. 3. Leader Yaw Angle in First Path under $H_{\infty}$ Controller

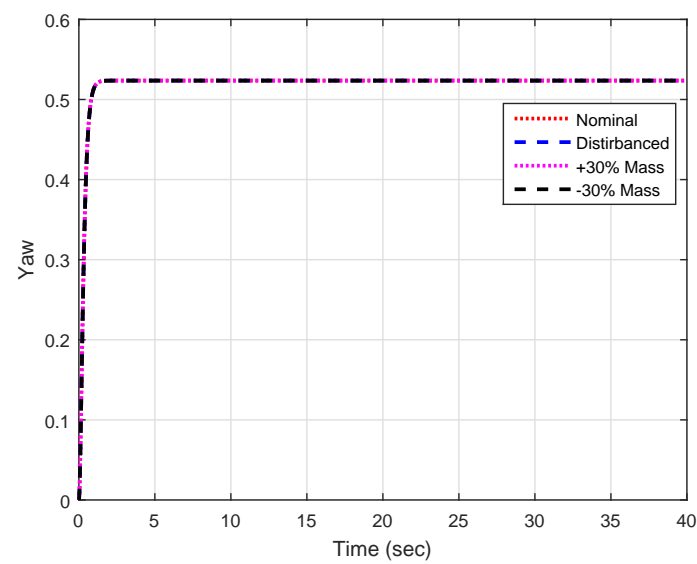

Fig. 4. Follower Yaw Angle in First Path under $H_{\infty}$ Controller



Fig. 5. Leader-Follower Formation in First Path under IBS Controller

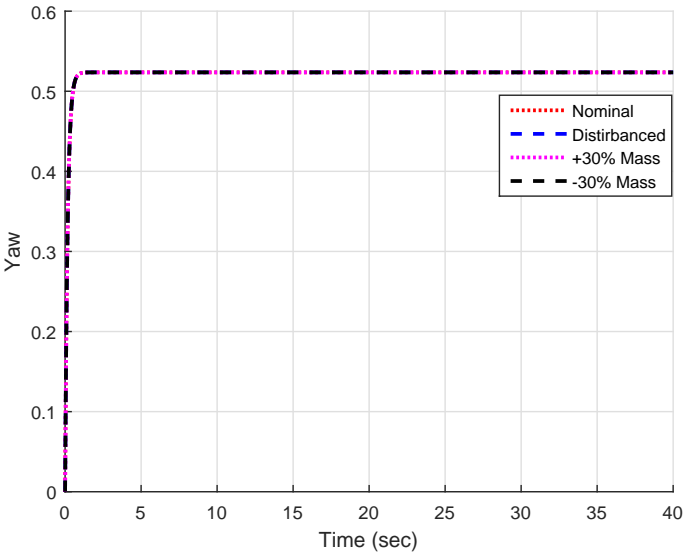

Fig. 6. Leader Yaw Angle in First Path under IBS Controller

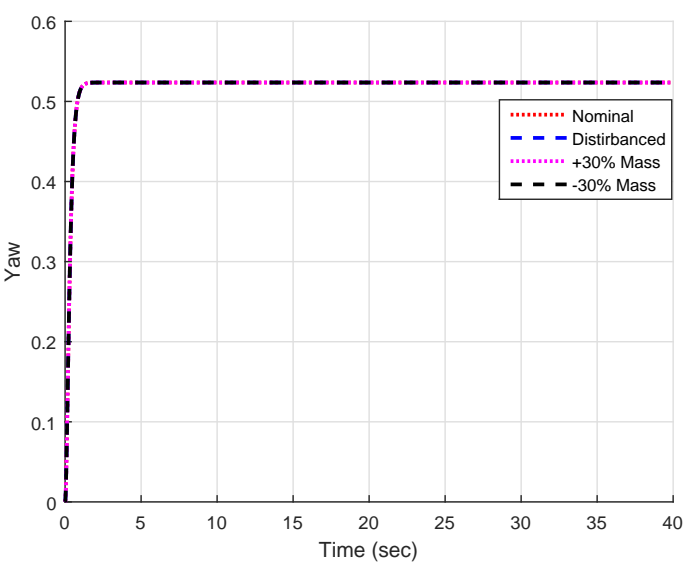

Fig. 7. Follower Yaw Angle in First Path under IBS Controller

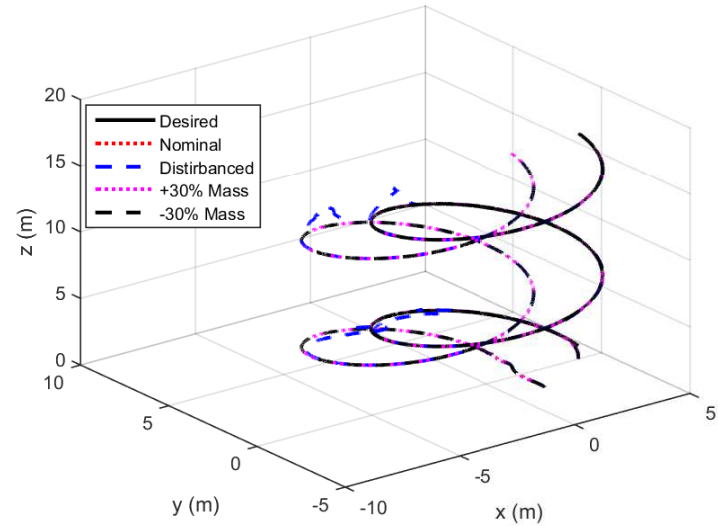

Fig. 8. Leader-Follower Formation in Second Path under $H_{\infty}$ Controller 


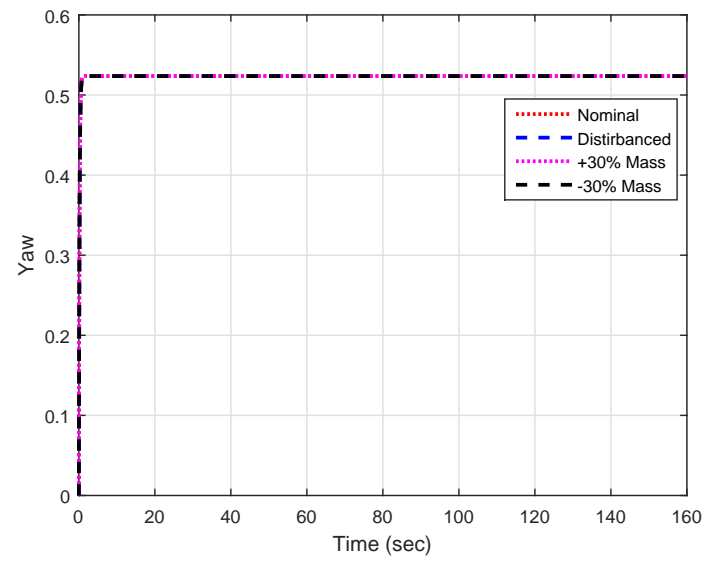

Fig. 9. Leader Yaw Angle in Second Path under $H_{\infty}$ Controller

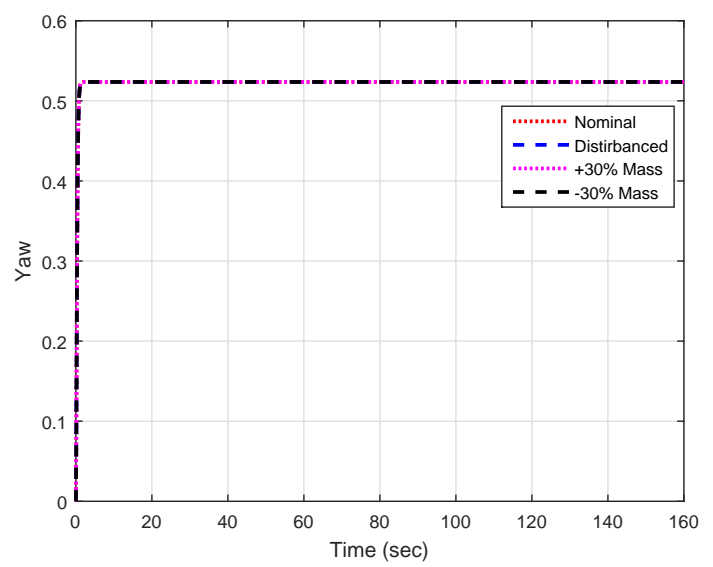

Fig. 10. Follower Yaw Angle in Second Path under $H_{\infty}$ Controller

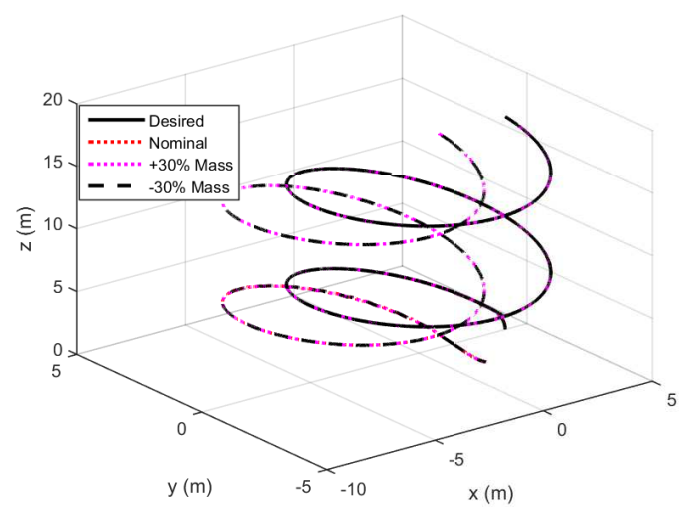

Fig. 11. Leader-Follower Formation in Second Path under IBS Controller

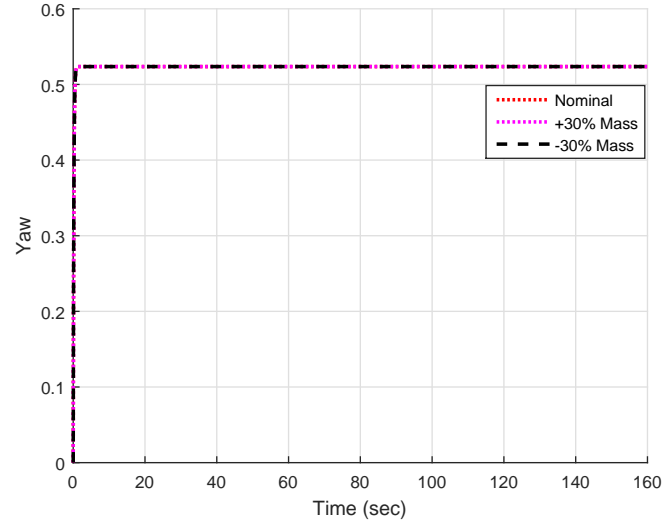

Fig. 12. Leader Yaw Angle in Second Path under IBS Controller

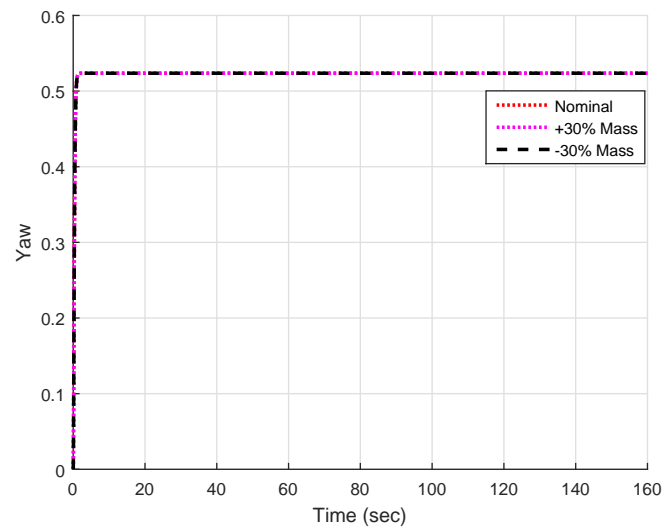

Fig. 13. Follower Yaw Angle in Second Path under IBS Controller
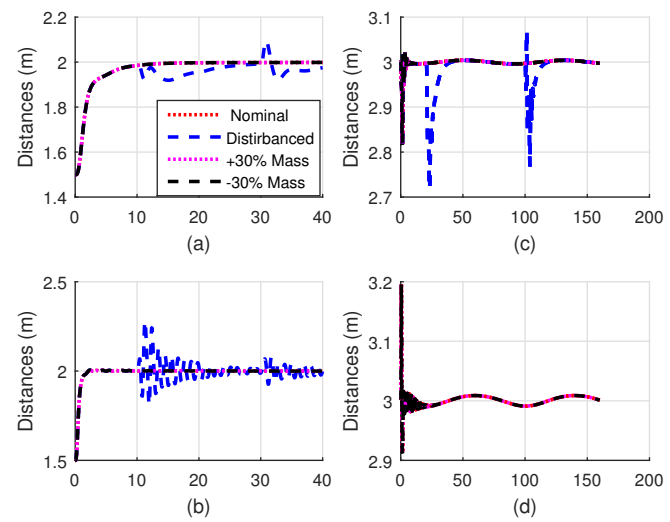

Fig. 14. The Distance Between the Leader and the Follower (a) in the First Path under $H_{\infty}$ Controller, (b) in the First Path under IBS Controller, (c) in the Second Path under $H_{\infty}$ Controller, (b) in the Second Path under IBS Controller 


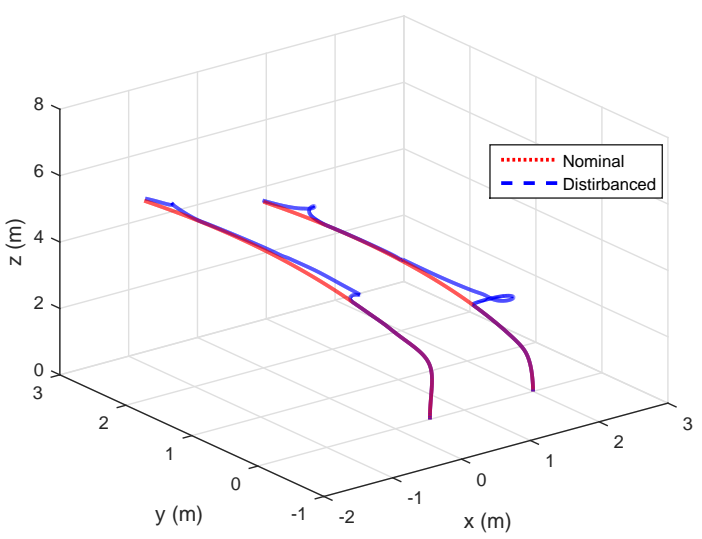

Fig. 15. Leader-Follower Formation in First Path under $H_{\infty}$ Controller with Leader Disturbance Only

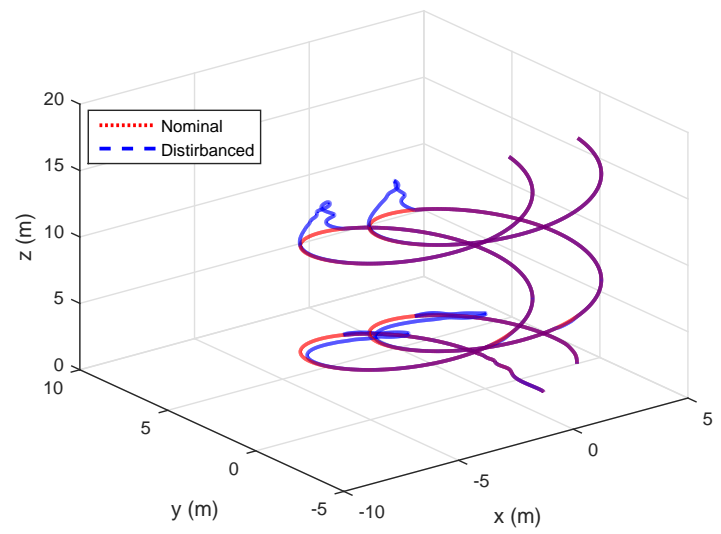

Fig. 16. Leader-Follower Formation in Second Path under $H_{\infty}$ Controller with Leader Disturbance Only

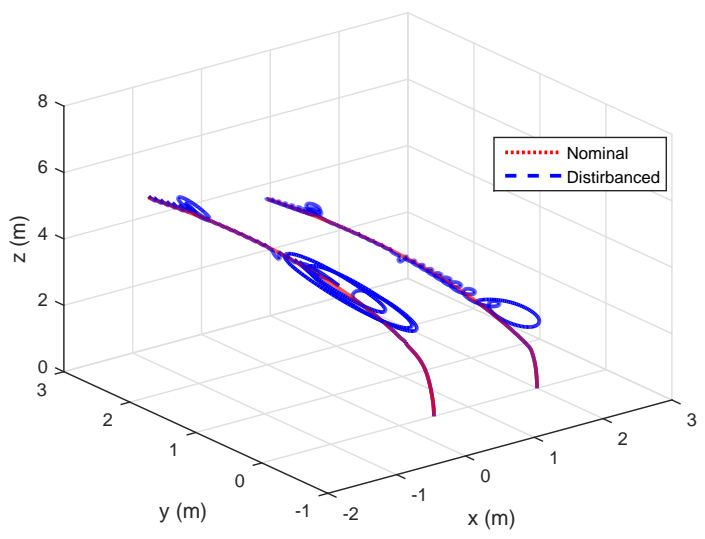

Fig. 17. Leader-Follower Formation in First Path under IBS Controller with Leader Disturbance Only

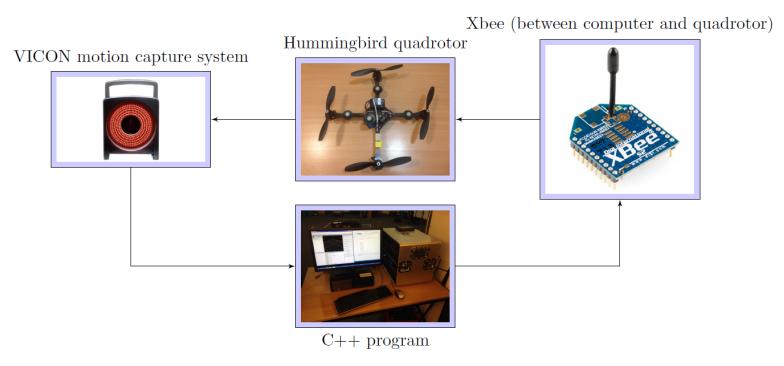

Fig. 18. Experimental Control Block Diagram

the desired follower position. Then the controller outputs were sent to the vehicles via the Xbees to the high level on-board microcontrollers which directly control the motors speed.

In the first test, both the leader and the follower took off to 0.5 metres and the formation controllers started from this point. Then the leader continued to climb to 1.5 metres, moved forward for 1 metre toward the $\mathrm{x}$-direction, drew a square of 2 metres side length and then landed. The follower followed the leader and maintained the desired distance between them $d=2$ metres, the desired incidence angle $\rho=\pi / 12$ and the desired bearing angle $\sigma=-\pi / 2$. In the second test, both the leader and the follower took off to 0.5 metres. Then the leader tracked a helical path of 2 metres circle diameter and 1.5 metres height, moved 1 metre towards the origin point and then landed. The follower tracked the leader and maintained the desired distance between them $d=2$ metres, the desired incidence angle $\rho=-\pi / 12$ and the desired bearing angle $\sigma=$ $-\pi / 2$. In the third test, both the leader and the follower took off to 0.5 metres. Then the leader tracked an eight-shaped path. The follower tracked the leader and maintained the desired distance between them $d=2$ metres, the desired incidence angle $\rho=-\pi / 12$ and the desired bearing angle $\sigma=-\pi / 3$.

The experimental trajectories of the first, second and third tests are shown in Figures 19 - 21, respectively, with the conditions (1) no disturbance, (2) force disturbance, and (3) $+20 \%$ mass. These conditions were applied to both the leader and the follower. Figure 22 illustrates the actual distance between the leader and the follower during the tests. The disturbances of the trajectories in these figures were caused by the external force exerted on the vehicles. These experimental results show that the follower successfully tracked the leader and maintained the distance, incidence and bearing angles between them with an acceptable error, less than 5 centimetres. The proposed controllers also show a good stability and robustness when considering the external disturbances and the mass change.

\section{CONCLUSIONS}

This paper presents a comparison between two different controllers to leader-follower formation control problem of quadrotors. The effect of the external disturbance and the model parameter uncertainties are considered. An $H_{\infty}$ suboptimal control approach to designing a state feedback controllers was proposed. The controller stability and robustness were analysed and a set of corresponding conditions was given. The 


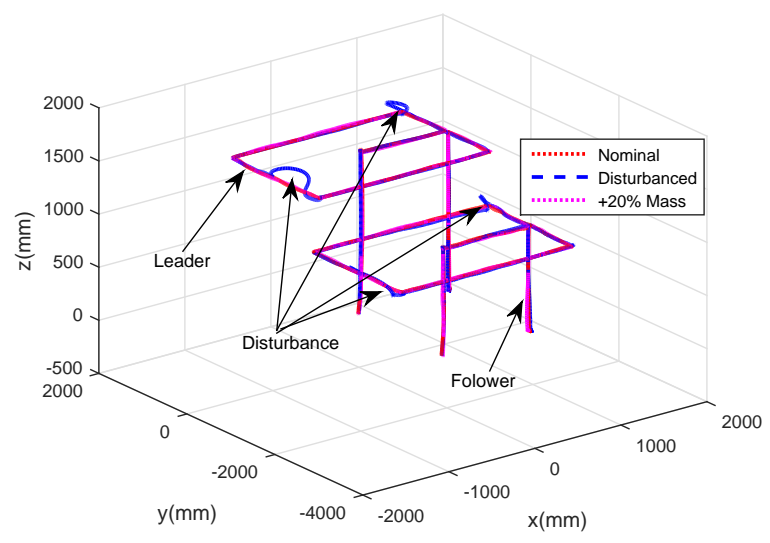

Fig. 19. Leader-Follower Formation in the Square Path Test

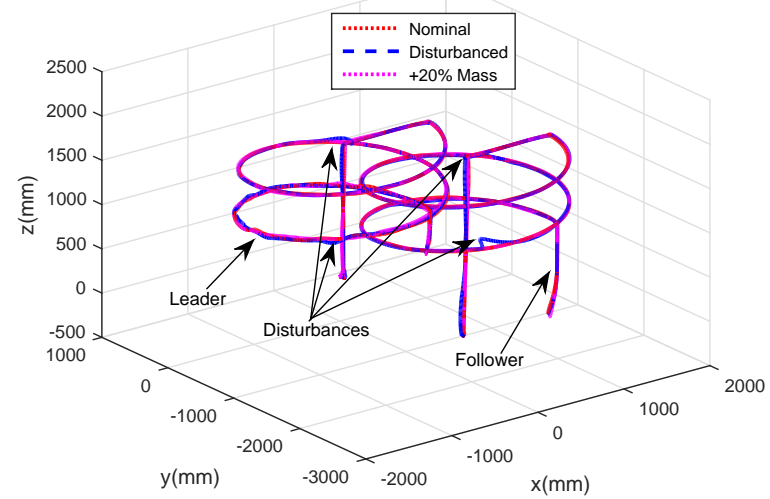

Fig. 20. Leader-Follower Formation in the Helical Path Test

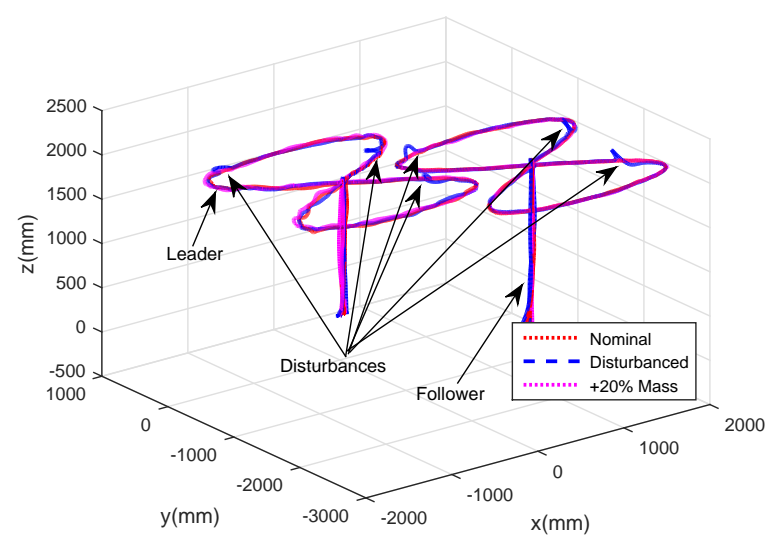

Fig. 21. Leader-Follower formation in the Eight Shape Test

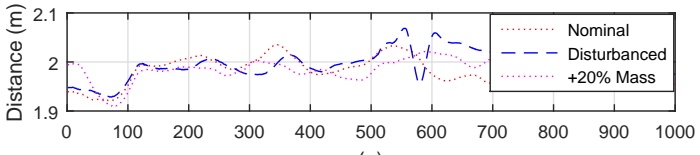

(a)

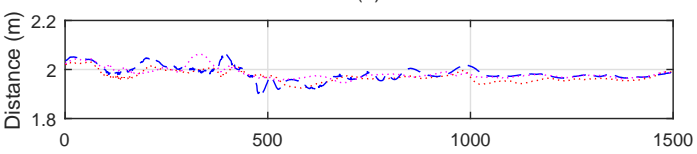

(b)

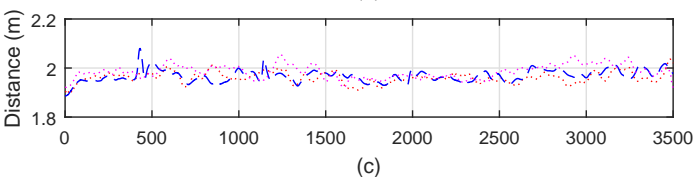

Fig. 22. The Distance Between the Leader and the Follower in (a) the Square Test, (b) the Helical Test, (c) the Eight Shape Test

second controller derived to compare with the proposed $H_{\infty}$ controller is integral backstepping controller. Both the controllers were tested in MATLAB simulater. Simulation results show that the proposed $H_{\infty}$ controller perform better than that of integral backstepping. The proposed $H_{\infty}$ controllers were tested on the vehicles via several flight scenarios with external disturbance and mass change consideration. Our next step work along this direction is to relax the two constant incidence and bearing angles to demonstrate how the formation performance can be improved with more followers.

\section{REFERENCES}

[1] S. Islam, P. X. Liu, and A. E. Saddik, "Robust control of fourrotor unmanned aerial vehicle with disturbance uncertainty," Industrial Electronics, IEEE Transactions on, vol. 62, no. 3, pp. 1563-1571, March 2015.

[2] J. Ferruz, V. M. Vega, A. Ollero, and V. Blanco, "Reconfigurable control architecture for distributed systems in the hero autonomous helicopter," Industrial Electronics, IEEE Transactions on, vol. 58, no. 12, pp. 53115318, December 2011.

[3] W. Ren, H. Chao, W. Bourgeous, N. Sorensen, and Y. Chen, "Experimental validation of consensus algorithms for multivehicle cooperative control," IEEE Transactions on Control Systems Technology, vol. 16, pp. 745-752, July 2008.

[4] H. Cai and J. Huang, "The leader-following consensus of multiple rigid spacecraft systems," in American Control Conference ACC '13, (Washington, DC, USA), pp. 824-829, 2013.

[5] T. Dierks and S. Jagannathan, "Neural network control of quadrotor UAV formations," in American Control Conference ACC '09, (Hyatt Regency Riverfront, St. Louis, MO, USA), pp. 2990-2996, 2009.

[6] A. Abdessameud, A. Tayebi, and I. G. Polushin, "Motion coordination of thrust-propelled underactuated vehicles in the presence of communication delays," in 19th IFAC Congress, (Cape Town, South Africa), pp. 3170-3175, 2014.

[7] D. Lee, "Distributed backstepping control of multiple thrust-propelled vehicles on balanced graph," in 18th IFAC Congress, (Milano, Italy), pp. 8872-8877, 2011.

[8] U. Pilz, A. Popov, and H. Werner, "Robust controller design for formation flight of quad-rotor helicopters," in Joint 48th IEEE Conference on Decision and Control and 28th Chinese Control Conference, (Shanghai, P.R. China), pp. 8322-8327, 2009.

[9] J. A. Guerrero, P. Castillo, and Y. Challal, "Quadrotors formation control a wireless medium access aware approach," Journal of Intelligent and Robotic Systems, vol. 4, pp. 221-231, 2013.

[10] J. Wang and M. Xin, "Integrated optimal formation control of multiple unmanned aerial vehicles," IEEE Transactions on Control Systems Technology, vol. 21, pp. 1731-1744, Sept 2013. 
[11] G. Regula and B. Lantos, "Formation control of quadrotor helicopters with guaranteed collision avoidance via safe path," Electrical Engineering and Computer Science, vol. 4, no. 2012, pp. 113-124, 2013.

[12] I. Bayezit and B. Fidan, "Distributed cohesive motion control of flight vehicle formations," Industrial Electronics, IEEE Transactions on, vol. 60, no. 12, pp. 5763-5772, December 2013.

[13] M. Saska, Z. Kasl, and L. Preucil, "Motion plannig and control of formations of micro aireal vehicles," in 19th IFAC Congress, (Cape Town, South Africa), pp. 1228-1233, 2014.

[14] A. T. Hafez, A. J. Marasco, S. N. Givigi, M. Iskandarani, S. Yousefi, and C. A. Rabbath, "Solving multi-uav dynamic encirclement via model predictive control," IEEE Transactions on Control Systems Technology, vol. 23, pp. 2251-2265, Nov 2015.

[15] A. Karimoddini, M. Karimadini, and H. Lin, "Decentralized hybrid formation control of unmanned aerial vehicles," arXiv preprint arXiv: 1403.0258, no. 1, 2014.

[16] L. C. A. Pimenta, G. A. S. Pereira, N. Michael, R. Mesquita, M. M. Bosque, L. Chaimowics, and V. Kumer, "Swarm coordination based on smoothed particle hydrodynamics technique," IEEE Transaction on Robotics, vol. 29, no. 2, pp. 383-399, 2013.

[17] M. Turpin, N. Michael, and V. Kumar, "Decentralized formation control with variable shapes for aerial robots," in 2012 IEEE International Conference on Robotics and Automation, (River Centre, Saint Paul, Minnesota, USA), pp. 23-30, 2012.

[18] M. Turpin, N. Michael, and V. Kumar, "Trajectory design and control for aggressive formation flight with quadrotors," Autonomous Robots, vol. 33, no. 1-2, pp. 143-156, 2012.

[19] M. Turpin, N. Michael, and V. Kumar, "CAPT: Concurrent assignment and planning of trajectories for multiple robots," The International Journal of Robotics Research, vol. 33, no. 1, pp. 98-112, 2014.

[20] A. Bemporad and C. Rocchi, "Decentralized hybrid model predictive control of a formation of unmanned aerial vehicles," in 18th IFAC World Congress, (Milano, Italy), 2011.

[21] A. Abdessameud and A. Tayebi, "Formation control of VTOL-UAVs," in Joint 48th IEEE Conference on Decision and Control and 28th Chinese Control Conference, (Shanghai, P.R. China), pp. 3454-3459, 2009.

[22] F. B. Choi and D. Lee, "Teleoperation control of formation among multiple under-actuated quadrotor UAVs," 2013 IEEE RO-MAN, The 22nd IEEE International Symposium on Robot and Human Interactive Communication, no. 3, pp. 306-307, 2013.

[23] R. Tjahjana and P. Sasongko, "Multi flying vehicle control for saving fuel," Applied Mathematical Sciences, vol. 8, no. 15, pp. 733-742, 2014.

[24] B. Yu, X. Dong, Z. Shi, and Y. Zhong, "Formation control for quadrotor swarm systems: Algorithms and experiments," in Control Conference (CCC), 2013 32nd Chinese, (Xi'an, China), pp. 7099-7104, July 2013.

[25] A. van der Schaft, " $L_{2}$-gain analysis of nonlinear systems and nonlinear state feedback $H_{\infty}$ control," IEEE Transactions on Automatic Control, vol. 37, no. 6, pp. 770-784, 1992.

[26] W. Jasim and D. Gu, "Robust path tracking control for quadrotors," in Robotica, Under Revision. 